\begin{tabular}{|l|}
\hline Summary \\
Adequacy \\
Alignment \\
Check lines 1-5 \\
Bones \\
Cartilage and joints \\
Zygomaticofrontal suture \\
Sinuses \\
Opacification \\
Air-fluid levels \\
Soft tissue \\
Swelling \\
Foreign bodies
\end{tabular}
This is the sixth in a series
articles describing current techniques in minimal access surgery. The articles have been written to inform nonspecialists of developments in this rapidly moving subject.

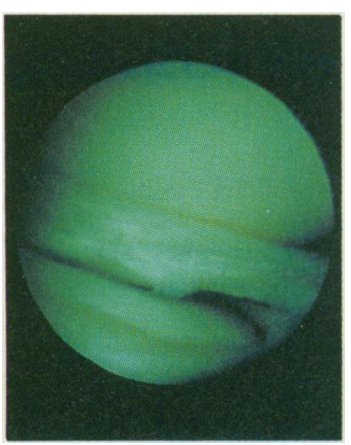

FIG 1-Torn medial meniscus in knee joint

\begin{abstract}
Addenbrooke's Hospital, Cambridge CB2 2 QQ Richard N Villar, consultant orthopaedic surgeon
\end{abstract}

$B M \mp 1994 ; 308: 51-3$

\section{Arthroscopy}

\author{
Richard N Villar
}

Normal anatomical structures can sometimes be mistaken for fractures. There are four common sites:

- Air in the oropharynx at the. angle of the mandible. If checked carefully this line will extend beyond the outer cortex of the mandible

- Calcification or ossification of the stylohyoid ligament projecting over or just behind the ascending ramus

- The hyoid bone shown over the posterior part of the horizontal ramus - The intervertebral spaces of the upper cervical vertebrae overlying the maxillae, simulating a LeFort 1 fracture, or over the mandibular symphysis, mimicking a dentoalveolar fracture.

Fractures of the mandible, particularly at the angle and the condyle, can appear undisplaced when seen in only one view. At least two views at right angles to each other are essential for full assessment-posteroanterior, Towne's, or lateral oblique.

The anterior mandible can be difficult to see in the orthopantomogram and lateral oblique projection because of superimposition of other structures. A lower occlusal view of the anterior mandible may therefore be useful in certain situations.

D W Hodgkinson is lecturer in emergency medicine, R E Lloyd is consultant maxillofacial surgeon, P A Driscoll is senior lecturer in emergency medicine, and D A Nicholson is consultant radiologist, Hope Hospital, Salford.

The line drawings were prepared by Mary Harrison, medical illustrator.

The ABC of Emergency Radiology has been edited by David Nicholson and Peter Driscoll.

\title{
Minimally Invasive Surgery
}

Arthroscopy has reduced the morbidity and period of hospitalisation associated with orthopaedic surgery and has increased the range of procedures that may be performed. From early operations on the knee it has expanded to include procedures for the shoulder, elbow, wrist, hip, ankle, and foot. For some joints the indications for surgery are clear, for others the clinical advantages are still being assessed. This expansion has also led to the recognition of complications, though the incidence is low. Specialist instrumentation has allowed a wide variety of operations previously needing open surgery to be carried out arthroscopically. The repertoire of arthroscopic procedures will undoubtedly continue to expand, and controlled studies are required to validate their efficacy, particularly in the management of degenerative joint diseases.

Arthroscopy has revolutionised orthopaedic surgery: combining diagnostic accuracy, low morbidity, and short length of hospitalisation, it is now one of the commonest orthopaedic procedures performed. As with all innovations, however, arthroscopy has had sceptics, and in 1937 Hustinx said of arthroscopy of the knee: "How can anyone venture to introduce a luminous object into the knee-joint in an effort to look between the articular surfaces, which cannot be separated...? This is quite impossible. Moreover, this procedure is more dangerous than exploratory arthrotomy."

The development of a rod lens system (designed by Professor Hopkins) surrounded by light conducting glass fibrils and enclosed in a rigid metal sheath led to the modern arthroscope. It soon became apparent that this versatile instrument had numerous applications. It was initially used for diagnostic purposes only, but instrumentation was developed to allow treatment of pathology as well. By the late 1970s arthroscopic operations had become established in the developed world. Early operations were mainly performed on the knee. Being mobile and easily accessible, it is particularly suitable for arthroscopy. Much was learnt about its anatomy and the natural history of knee conditions.

Orthopaedic surgeons and their patients soon appreciated the advantages of such keyhole surgery on the knee, and other joints were investigated. Burman had paved the way for this in 1931 by looking at many different joints in cadavers. ${ }^{2}$ Arthroscopy may now be performed in many joints including the shoulder, elbow, wrist, ankle, hip, and even the first metatarsophalangeal joint.

\section{Knee arthroscopy}

The knee is still the most frequently arthroscoped joint, although the advent of magnetic resonance imaging has reduced the need for diagnostic knee arthroscopy. Much recent research has been performed to relate the findings of magnetic resonance imaging to those of arthroscopy ${ }^{3}$ : in general, a negative scan is probably correct but a positive scan may not be. Arthroscopy should only be performed, however, if the symptoms suggest it.

Though the need for diagnostic knee arthroscopy recedes, the range of operative procedures available increases. It is now possible to perform arthroscopic reconstruction of the anterior cruciate ligament, meniscal repair, fixation of a tibial plateau fracture, and assessment of a painful total knee replacement as well as more established techniques such as meniscectomy (fig 1), synovectomy, and retrieval of loose bodies. 


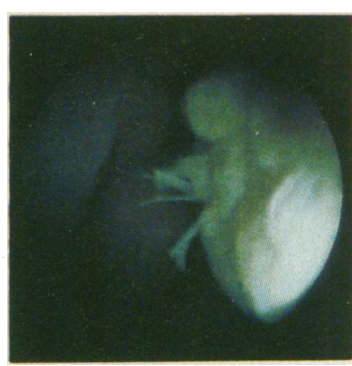

FIG 2-Large loose body in hip joint
Another major use of knee arthroscopy is the management of osteoarthritis. Despite many studies we still do not know whether a patient's pain can be significantly reduced by arthroscopic lavage alone ${ }^{4}$ or debridement. Current opinion suggests that arthroscopic lavage may be able to help in some cases, but the precise clinical indications for it have still not been defined. ${ }^{5}$

\section{Shoulder arthroscopy}

The arthroscopic anatomy of the shoulder only became clearly defined in the mid- 1980 s. $^{6}$ Operations may be performed under general or local anaesthesia or on an outpatient basis. Operations suitable for arthroscopy include the removal of loose fragments, debridement of degenerative lesions, repair of instability, drainage of sepsis, and correction of impingement syndromes. ${ }^{7}$ Its use for frozen shoulder has still not clarified the precise cause of this condition. ${ }^{8}$ Complications, fortunately uncommon, have been recognised and may include damage to the brachial plexus or axillary nerve.

\section{Elbow arthroscopy}

Elbow arthroscopy is a relatively new procedure, and reports are largely confined to small, statistically unsupported studies. Suitable operations include the diagnosis and removal of loose bodies, removal of intra-articular plica, management of osteochondritis, and repair of instability of the joint. Post-traumatic arthritis, inflammatory arthritis, and primary degenerative disease are also common indications for the operation.

Because of the joint's bony anatomy, arthroscopy of the elbow is technically more demanding than arthroscopy of the shoulder or knee. Overall, elbow arthroscopy can produce diagnostic benefits in more than $60 \%$ of patients and symptomatic improvement in over $70 \%,{ }^{9}$ though the best results are obtained after removal of loose bodies. Patients for whom all preoperative investigations are normal but who still have pain are unlikely to benefit. Experience suggests that such patients' clinical managment is not altered by arthroscopy. Complications can be expected in $10 \%$ of cases, though these are rarely major. However, the promixity of several major neurovascular structures, particularly the radial nerve, suggests that arthroscopy of the elbow is not suitable for an occasional operator.

\section{Hip arthroscopy}

In 1931 Burman had stated: "It is manifestly impossible to insert a needle between the head of the femur and the acetabulum." "This was the prevalent view for several decades, ${ }^{10}$ but in the early 1980 s occasional reports appeared on the use of hip arthroscopy in certain specific and unusual situations. It was advocated as a means of assisting in reducing a dislocated total hip replacement, ${ }^{11}$ as a method of assessing paediatric hip disorders, ${ }^{12}$ and even as a suitable approach for removing a bullet lodged in the joint. ${ }^{13}$ In these cases the surgeons usually had a standard orthopaedic traction table, often applying more than $25 \mathrm{~kg}$ of pull to distract the joint.

Since these early reports, the indications for the procedure have become clearer. Hip arthroscopy can be used for the management of the torn acetabular labrum, ${ }^{14}$ removal of loose bodies (fig 2), synovectomy, trauma, and other conditions. The list grows daily. The main test for hip arthroscopy, however, will lie in its effect on the osteoarthritic hip, particularly for younger patients. Though femoral and pelvic osteotomy and even joint replacement have been advocated for the young degenerate hip joint, most would agree that such options are major procedures. No controlled studies on the effects of hip arthroscopy on the degenerate hip have been performed, but recent reports suggest that a significant improvement can be obtained, certainly for at least two years. ${ }^{15}$ Patients aged over 55 and those with severe osteoarthritis do not respond well, and hip arthroscopy is best avoided in such cases.

Arthroscopy of the hip has diagnostic advantages over any other method of imaging the hip joint currently available: Glick reported that it allowed a diagnosis to be made in $40 \%$ of cases of painful hips where all prior investigations had been normal. ${ }^{16} \mathrm{~A}$ recent prospective study found large discrepancies between the findings of magnetic resonance imaging and those of arthroscopy. Imaging appears to have difficulty with the identification of partial thickness defects in the articular surface and in the identification of cartilaginous loose bodies (D Edwards et al, first international symposium on hip arthroscopy, Cambridge, 1992).

Introducing an arthroscope into the hip joint requires great care. Despite the application of great force, the ball and socket nature of the joint and its natural intraarticular vacuum mean that distraction is minimal until air or fluid has been introduced into the joint. When this is achieved distraction of $3-4 \mathrm{~cm}$ is normal, allowing easy passage of a standard arthroscope of 4.5 $\mathrm{mm}$ diameter. The procedure is best performed under the guidance of an image intensifier so that the arthroscope can be accurately placed in the deep seated joint. Standard knee arthroscopic equipment can be used, but it may not be long enough to reach the innermost depths of the acetabulum of an obese or muscular patient. Extended arthroscopes and operating instruments are now available to cope with this.

Few complications have been reported, but the total world experience of hip arthroscopy is probably little more than 750 cases. There are isolated reports of transient neural damage, breakage of instruments, articular scuffing, and pressure sores from prolonged distraction. The complex procedure is time consuming: if all goes well a hip arthroscopy will take at least 45 minutes, and some have reported operating times in excess of two hours. The development of specialist hip distractors (fig 3) and cannulated instrumentation to gain easy access to the joint over a guide wire should significantly reduce operating times in the near future. The scene is now set for major developments in hip arthroscopy.

\section{Ankle and foot arthroscopy}

The ankle and subtalar and metatarsophalangeal joints are accessible to arthroscopy. This may be performed under general or local anaesthesia and now forms part of specialised orthopaedic and podiatric

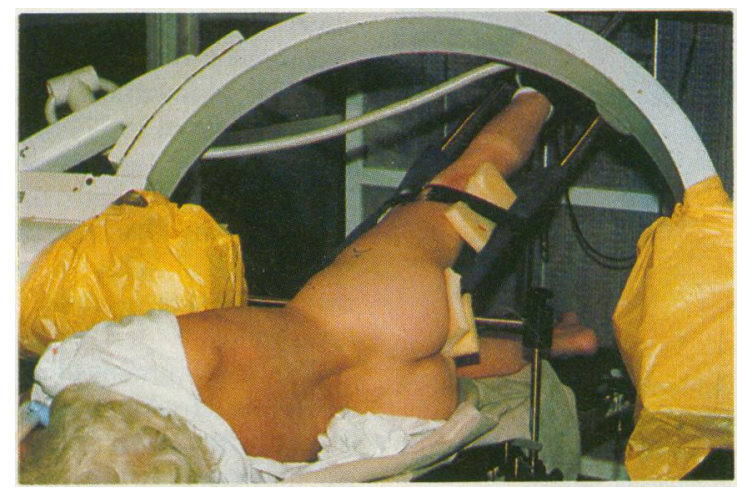

FIG 3-Specialist hip distractors should make arthroscopic access easier 


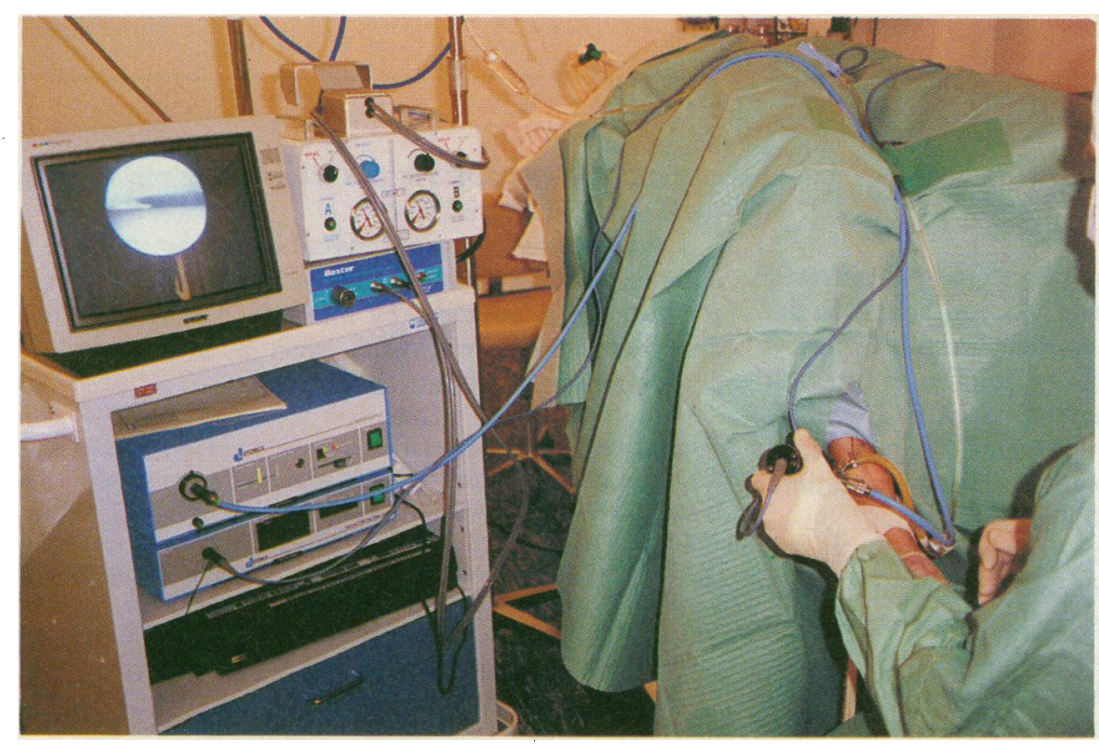

FIG 4-Anterior approach to ankle arthroscopy practice $^{17}$ and may occasionally be performed as an office procedure. The ankle can be approached anteriorly (fig 4) or posteriorly, and distraction can be provided by an external fixator or by special straps that allow the foot to be pulled down by the surgeon during an operation. Loose bodies, cartilaginous defects, impingement lesions, and synovitis are some of the indications for the procedure. Indeed, arthroscopic ankle arthrodesis is now an accepted procedure for the painful end stage ankle joint in certain cases.

Arthroscopy of the subtalar joint has limited applications, largely being confined to managing degenerative joint disease and synovitis. Synovectomy or debridement of irregular joint surfaces can be performed, with the approach being made either posteriorly or through the sinus tarsi. The great toe and smaller metatarsophalangeal joints are also amenable to arthroscopic procedures, particularly when radiographic changes are minimal despite establishment of the pain of synovitis or degenerative change. A standard, $4.5 \mathrm{~mm}$ diameter arthroscope is impractical for these smaller joints. It either does not fit or causes extensive articular scuffing. Smaller arthroscopes, as little as $1.7 \mathrm{~mm}$ in diameter, are available. They are based on a gradient index (GRIN) lens system as the Hopkins rod lens system is inappropriate for such small sizes.

\section{Complications}

Compared with many other surgical procedures the risks of arthroscopy are low. Complications do occur, however, though such problems were not formally identified until $1978 .{ }^{18}$ An overall complication rate of $1.8 \%$, based on 1988 data, has been confirmed by information from the Complications Committee of the Arthroscopy Association of North America. ${ }^{19}$ This figure is double that quoted by a similar survey two years earlier. Reports from the United Kingdom are few, though a rate of $0.02 \%$ for knee arthroscopy has recently been reported. ${ }^{20}$ There is, however, an obvious concern that the frequency of complications will

The risk of infection is small, possibly less than one in 1000 , but can increase substantially depending on the complexity of the procedure performed. For example, arthroscopic reconstruction of the anterior cruciate ligament, which is increasingly being performed, can be associated with rates of infection of up to $3.6 \%$. The frequency of complications is also governed by the joint being operated on: complications are less likely during arthroscopy of the knee, a largely superficial joint, than during shoulder arthroscopy. The tighter, less distractible joints such as the hip, ankle, and elbow are more difficult to instrument than the knee and shoulder, and scuffing of articular cartilage is thus a genuine risk. There is as yet little evidence on the long term consequences of scuffing, and every effort should be made to minimise such damage.

\section{Future developments}

Despite the earlier doubts of orthopaedic surgeons, arthroscopy is here to stay. Few joints remain to be explored, and the operative techniques possible for the joints now established continue to expand. Because the complication rate is so low, regardless of which joint is involved, there is a tendency to perform procedures more readily. The 1990 s will probably show increasing numbers of carefully controlled studies that will establish the clinical efficacy of the numerous arthroscopic procedures now possible. Such information is particularly needed in the management of degenerative joint disease: it is now technically possible to remove loose articular flaps and debris arthroscopically, but, whether such techniques alter the long term outcome of the disease is, as yet, unknown.

1 Eikelaar HR. Historical review. In: Arthroscopy of the knee. The Netherlands: Royal United Printers Hoitsema BV, 1980:6-12.

2 Burman MS. Arthroscopy or the direct visualisation of joints: an experimental cadaver study. F Bone foint Surg 1931;8:669-95.

3 Boeree NR, Watkinson AF, Ackroyd CE, Johnson C. Magnetic resonance imaging of meniscal and cruciate injuries of the knee. $f$ Boint Surg $(B r)$ 1991;73:452-7.

4 Jackson RW. Arthroscopic treatment of degenerative arthritis. In: McGinty JB, ed. Operative arthroscopy. New York: Raven Press, 1991:319-23.

5 Dandy DJ. Editorial: Arthroscopic debridement of the knee for osteoarthritis.

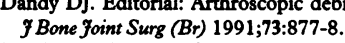

6 Matthews LS, Terry G. Shoulder anatomy for the arthroscopist. Arthroscopy 1985;1:83.

7 Wiley AM. Arthroscopy of the shoulder and the subacromial bursa. In Watson M. ed. Surgical disorders of the shoulder. Edinburgh: Churchil Livingstone, 1991:157-64.

8 Wiley AM. Arthroscopic appearances of frozen shoulder. Arthroscopy 1991;7: $138-43$.

9 O'Driscoll SW, Morrey BF. Arthroscopy of the elbow: diagnostic and therapeutic benefits and hazards. $\mathcal{F}$ Bone foint Surg ( $\mathrm{Am}$ ) 1992;74:84-94.

10 Villar RN. The history of hip arthroscopy. In: Hip arthroscopy. Oxford: Butterworth-Heinemann, 1992:1-5.

11 Shifrin LZ, Reis ND. Arthroscopy of a dislocated hip replacement. Clin Orthop 1980;146:213-4.

12 Gross RH. Arthroscopy in hip disorders in children. Orthop Rev 1977;6:43-9.

13 Goldman A, Minkoff J, Price A, Krinick R A posterior arthroscopic approach

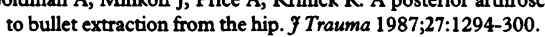

14 Ikeda T, Awaya G, Okada Y, Tada $\mathrm{H}$. Torn acetabular labrum in youn patients. $f$ Bone foint Surg (Br) 1988;70:13-6.

15 Villar RN. Arthroscopic debridement of the hip. 7 Bone foint Surg (Br) 1991;73 (suppl II):922-6.

16 Glick JM. Hip arthroscopy. In: McGinty JB, ed. Operative arthroscopy. New York: Raven, 1991:663-76.

17 Lundeen RO. Arthroscopic techniques of the ankle and foot. In: Manual of ankle and foot arthroscopy. Edinburgh: Churchill Livingstone, 1992:45-56.

18 Jackson RW, Wortzmann G. Complications of invasive diagnostic procedures. In: Epps CH Jr, ed. Complications in orthopaedic surgery. Vol 1. Philadelphia: Lea and Febiger, 1978:70-2.

19 Small NC. General overview of complications in arthroscopic surgery. In: Sherman $\mathrm{OH}$, Minkoff J, eds. Current management of complications in orthopaedics. Arthroscopic surgery. Baltimore: Williams and Wilkins, 1990:1-9.

20 Bamford DJ, Noble J, Davies DRA. Avoidable complications of arthroscopic surgery. F Bone foint Surg (Br) 1992;74 (suppl ID):328. 